\title{
Correction to: Gout and healthcare utilization and complications after hip arthroplasty: a cohort study using the US National Inpatient Sample (NIS)
}

\author{
Jasvinder A. Singh ${ }^{1,2,3,4}$ (D) John D. Cleveland ${ }^{2,4}$ \\ Published online: 29 July 2019 \\ (C) International League of Associations for Rheumatology (ILAR) 2019
}

\section{Correction to: Clinical Rheumatology (2019) 38:1213-1216 https://doi.org/10.1007/s10067-019-04434-3}

The above article originally published with an error present in Table 1. The data "Home" on the first column of the Table 1 should be interchange with the previous data "Inpatient facility $\uparrow "$. The corrected Table is shown as follows:

The online version of the original article can be found at https://doi.org/ 10.1007/s10067-019-04434-3

Jasvinder A. Singh

Jasvinder.md@gmail.com

John D. Cleveland

jcleveland@uabmc.edu

1 Medicine Service, VA Medical Center, 700 19th St S, Birmingham, AL 35233, USA

2 Department of Medicine at School of Medicine, University of Alabama at Birmingham, 1720 Second Ave. South, Birmingham, AL 35294-0022, USA

3 Division of Epidemiology, University of Alabama at Birmingham, 1720 Second Ave. South, Birmingham, AL 35294-0022, USA

4 University of Alabama at Birmingham, Faculty Office Tower 805B, 510 20th Street S, Birmingham, AL 35294-0022, USA 
Table 1 Demographic and other cohort characteristics

\begin{tabular}{|c|c|c|c|}
\hline & $\begin{array}{l}\text { All Cohort } \\
(\mathrm{N}=4116485)^{*}\end{array}$ & $\begin{array}{l}\text { No Gout } \\
(\mathrm{N}=4012387)^{*}\end{array}$ & $\begin{array}{l}\text { Gout } \\
(\mathrm{N}=104098)^{*}\end{array}$ \\
\hline Age, Mean (SE); median & $65.2(0.04) ; 65.9$ & $65.5(0.04) ; 66.0$ & $67.0(0.09) ; 67.0$ \\
\hline \multicolumn{4}{|l|}{ Age category } \\
\hline$<50$ & $449,642(10.9 \%)$ & $442,224(11.0 \%)$ & $7418(7.1 \%)$ \\
\hline $50-64$ & $1,364,821(33.2 \%)$ & $1,330,919(33.2 \%)$ & $33,902(32.6 \%)$ \\
\hline $65-79$ & $1,732,014(42.1 \%)$ & $1,683,378(42.0 \%)$ & $48,636(46.7 \%)$ \\
\hline$\geq 80$ & $566,521(13.8 \%)$ & $552,389(13.8 \%)$ & $14,132(13.6 \%)$ \\
\hline \multicolumn{4}{|l|}{ Gender } \\
\hline Female & $2,330,188(56.6 \%)$ & $2,303,984(57.4 \%)$ & $26,204(25.2 \%)$ \\
\hline Male & $1,776,722(43.2 \%)$ & $1,698,847(42.3 \%)$ & $77,875(74.8 \%)$ \\
\hline \multicolumn{4}{|l|}{ Race } \\
\hline White & $2,882,040(70.0 \%)$ & $2,807,827(70.0 \%)$ & $74,213(71.3 \%)$ \\
\hline Black & $225,772(5.5 \%)$ & $217,805(5.4 \%)$ & $7967(7.7 \%)$ \\
\hline Hispanic & $104,385(2.5 \%)$ & $102,364(2.6 \%)$ & $2021(1.9 \%)$ \\
\hline Other/Missing & $904,234(22.0 \%)$ & $884,338(22.0 \%)$ & $19,896(19.1 \%)$ \\
\hline \multicolumn{4}{|l|}{ Primary Diagnosis } \\
\hline Rheumatoid arthritis & $29,173(0.7 \%)$ & $28,946(0.7 \%)$ & $227(0.2 \%)$ \\
\hline Avascular necrosis & $285,623(6.9 \%)$ & $277,134(6.9 \%)$ & $8489(8.2 \%)$ \\
\hline $\mathrm{OA}$ & $3,447,224(83.7 \%)$ & $3,357,983(83.7 \%)$ & $89,241(85.7 \%)$ \\
\hline Other & $354,307(8.6 \%)$ & $348,167(8.7 \%)$ & $6140(5.9 \%)$ \\
\hline Fracture & $117(0.0 \%)$ & $117(0.0 \%)$ & $0(0.0 \%)$ \\
\hline \multicolumn{4}{|l|}{ Hospital Location/Teaching } \\
\hline Rural & $444,188(10.8 \%)$ & $433,750(10.8 \%)$ & $10,438(10.0 \%)$ \\
\hline Urban & $1,722,391(41.8 \%)$ & $1,680,203(41.9 \%)$ & $42,188(40.5 \%)$ \\
\hline Urban Teaching & $1,939,989(47.1 \%)$ & $1,888,827(47.1 \%)$ & $51,162(49.1 \%)$ \\
\hline \multicolumn{4}{|l|}{ Insurance } \\
\hline Medicaid & $138,809(3.4 \%)$ & $136,302(3.4 \%)$ & $2507(2.4 \%)$ \\
\hline Medicare & $2,234,674(54.3 \%)$ & $2,174,154(54.2 \%)$ & $60,520(58.1 \%)$ \\
\hline Other & $102,276(2.5 \%)$ & $100,185(2.5 \%)$ & $2091(2.0 \%)$ \\
\hline Private & $1,600,829(38.9 \%)$ & $1,562,620(38.9 \%)$ & $38,209(36.7 \%)$ \\
\hline Self & $32,308(0.8 \%)$ & $31,697(3.0 \%)$ & $611(0.6 \%)$ \\
\hline \multicolumn{4}{|l|}{ Income Category } \\
\hline $0-25$ th percentile & $653,243(15.9 \%)$ & $635,111(15.8 \%)$ & $18,132(17.4 \%)$ \\
\hline 25-50th percentile & $1,009,677(24.5 \%)$ & $984,542(24.5 \%)$ & $25,135(24.1 \%)$ \\
\hline 50-75th percentile & $1,086,954(26.4 \%)$ & $1,059,575(26.4 \%)$ & $27,379(26.3 \%)$ \\
\hline 75-100th percentile & $1,285,855(31.2 \%)$ & $1,254,558(31.3 \%)$ & $31,297(30.1 \%)$ \\
\hline \multicolumn{4}{|l|}{ Hospital Bed size } \\
\hline Small & $685,209(16.6 \%)$ & $667,201(16.6 \%)$ & $18,008(17.3 \%)$ \\
\hline Medium & $1,037,561(25.2 \%)$ & $1,010,906(25.2 \%)$ & $26,655(25.6 \%)$ \\
\hline Large & $2,383,797(57.9 \%)$ & $2,324,673(57.9 \%)$ & $59,124(56.8 \%)$ \\
\hline \multicolumn{4}{|l|}{ Hospital Region } \\
\hline Northeast & $818,700(19.9 \%)$ & $797,318(19.9 \%)$ & $21,382(20.5 \%)$ \\
\hline Midwest & $1,089,883(26.5 \%)$ & $1,062,567(26.5 \%)$ & $27,316(26.2 \%)$ \\
\hline South & $1,358,856(33.0 \%)$ & $1,323,743(33.0 \%)$ & $35,113(33.7 \%)$ \\
\hline West & $849,046(20.6 \%)$ & $828,759(20.7 \%)$ & $20,287(19.5 \%)$ \\
\hline \multicolumn{4}{|c|}{ Annual Hospital THA Volume } \\
\hline$<25$ & $486,120(11.8 \%)$ & $472,939(11.8 \%)$ & $13,086(12.5 \%)$ \\
\hline 26 to 100 & $1,461,615(35.5 \%)$ & $1,425,450(35.5 \%)$ & $25,980(24.8 \%)$ \\
\hline
\end{tabular}


Table 1 (continued)

\begin{tabular}{|c|c|c|c|}
\hline & $\begin{array}{l}\text { All Cohort } \\
(\mathrm{N}=4116485)^{*}\end{array}$ & $\begin{array}{l}\text { No Gout } \\
(\mathrm{N}=4012387)^{*}\end{array}$ & $\begin{array}{l}\text { Gout } \\
(\mathrm{N}=104098)^{*}\end{array}$ \\
\hline 101 to 200 & $1,051,561(25.5 \%)$ & $1,025,560(25.6 \%)$ & $36,312(34.7 \%)$ \\
\hline$>200$ & $1,117,189(27.1 \%)$ & $1,087,744(27.1 \%)$ & $29,414(28.1 \%)$ \\
\hline \multicolumn{4}{|l|}{ Deyo-Charlson Score } \\
\hline 0 & $2,193,575(53.3 \%)$ & $2,151,940(53.6 \%)$ & $41,635(40.0 \%)$ \\
\hline 1 & $926,286(22.5 \%)$ & $902,768(22.5 \%)$ & $23,518(22.6 \%)$ \\
\hline$\geq 2$ & $996,624(24.2 \%)$ & $957,679(23.9 \%)$ & $38,945(37.4 \%)$ \\
\hline \multicolumn{4}{|c|}{ Post-THA Healthcare Utilization \& In-hospital Complications } \\
\hline Infection & $7592(0.2 \%)$ & $7437(0.2 \%)$ & $155(0.1 \%)$ \\
\hline Revision & $17,932(0.4 \%)$ & $17,552(0.4 \%)$ & $380(0.4 \%)$ \\
\hline Transfusion & $937,803(22.8 \%)$ & $915,245(22.8 \%)$ & $22,558(21.7 \%)$ \\
\hline Gout & $104,098(2.5 \%)$ & - & $104,098(100.0 \%)$ \\
\hline \multicolumn{4}{|l|}{ Discharge Status } \\
\hline Home & $2,448,107(59.5 \%)$ & $2,386,634(59.5 \%)$ & $61,473(59.1 \%)$ \\
\hline Inpatient facility $\dagger$ & $1,649,102(40.1 \%)$ & $1,606,861(40.0 \%)$ & $42,241(40.6 \%)$ \\
\hline Length of Stay, Mean (SE); median & $3.71(0.01) ; 2.74$ & $3.73(0.01) ; 2.75$ & 3.67 (0.02); 2.69 \\
\hline \multicolumn{4}{|l|}{ Length of Stay category } \\
\hline$\leq 3$ & $2,499,883(60.7 \%)$ & $2,434,036(60.7 \%)$ & $65,847(63.3 \%)$ \\
\hline$>3$ & $1,616,602(39.3 \%)$ & $1,578,351(39.3 \%)$ & $38,251(36.7 \%)$ \\
\hline Died during hospitalization & $8889(0.2 \%)$ & $8737(0.8 \%)$ & $153(0.1 \%)$ \\
\hline
\end{tabular}

*U.S. National estimates were based on the following in the 20\% NIS sample: All, N=855634; no gout, N=834115; gout, N=21519; N (\%), unless specified otherwise

${ }^{\dagger}$ Inpatient facility included short- or long-term care hospital, skilled nursing facility (SNF), intermediate care facility, or a certified nursing facility

Publisher's note Springer Nature remains neutral with regard to jurisdictional claims in published maps and institutional affiliations. 